\title{
Mesenchymal Cells Isolated after Acute Lung Injury Manifest an Enhanced Proliferative Phenotype
}

\author{
Baruch Chen, *,* Vitaly Polunovsky, * James White," Bruce Blazar, " Raouf Nakhleh," \\ Jose Jessurun," Mark Peterson, * and Peter Bitterman * \\ University of Minnesota School of Medicine, * Department of Medicine, ${ }^{\S}$ Department of Pediatrics, "Department of Laboratory Medicine \\ and Pathology, Minneapolis, Minnesota 55455; and ${ }^{\ddagger}$ Sapir Medical Center, Kfar Saba 44281 Israel
}

\begin{abstract}
After acute lung injury, mesenchymal cells migrate into the alveolar airspace where they proliferate and deposit connective tissue macromolecules. Early in the disease process, inflammatory cell-derived trophic factors modulate these mesenchymal cell functions. However, in those patients who die, even as the inflammatory response abates, the fibroproliferative response continues, resulting in extensive intraalveolar fibrosis. We therefore hypothesized that lung mesenchymal cells obtained from individuals dying with acute alveolar fibrosis would manifest an enhanced proliferative capacity that was independent of persistent exogenous signals. To examine this hypothesis, the in vitro growth properties of mesenchymal cells prepared from patients dying with acute lung injury $(n=3)$ were analyzed in defined medium and compared with those of mesenchymal cells similarly prepared from patients dying with histologically normal lungs $(n=3)$. Isolates were characterized as mesenchymal cells by using morphological and immunohistochemical criteria. In accord with the hypothesis, mesenchymal cells isolated from lung-injured patients doubled within $3 \mathrm{~d}$ in the complete absence of exogenous peptide growth factors, reaching a saturation density of $\sim 15 \times 10^{3}$ cells $/ \mathrm{cm}^{2}$. As expected, lung mesenchymal cells from normal individuals failed to significantly increase in number. Consistent with this proliferative phenotype, the immediate early cell division cycle genes $c$-fos and $c$-jun were constitutively expressed in each cell strain prepared from injured lungs, but not in those from control lungs. The observed proliferative phenotype was stable through the fifth subcultivation of the cells. Despite these proliferative properties, three separate criteria indicated the mesenchymal cells from injured lungs were not transformed: normal karyotype; finite lifespan in vitro (9-10 subcultivations); and inability to disseminate in mice with severe combined immunodeficiency. These data support the hypothesis that mesenchymal cells manifest an enhanced proliferative state after acute lung injury. (J. Clin. Invest. 1992.90:1778-1785.) Key words: fibroblasts • lung injury - mesenchymal cells • proliferation
\end{abstract}

Address reprint requests to Peter Bitterman, M. D., Pulmonary Division, Department of Medicine, UMHC Box 276, University of Minnesota, 420 Delaware Street Southeast, Minneapolis, MN 55455.

Received for publication 13 January 1992 and in revised form 2 April 1992.

J. Clin. Invest.

(C) The American Society for Clinical Investigation, Inc.

0021-9738/92/11/1778/08 \$2.00

Volume 90, November 1992, 1778-1785

\section{Introduction}

The clinical outcome of patients after acute lung injury is critically dependent on the biological response of lung mesenchymal cells. Among those patients dying after acute lung injury, prompt and orderly reconstitution of alveolar anatomy fails to occur. Instead, a rapidly progressive fibroproliferative response ensues in which lung mesenchymal cells migrate into the airspace, proliferate, and deposit connective tissue $(1,2)$. The inability to control this fibroproliferative response results in obliteration of the alveolar airspace and failure of gas exchange. In the seminal stages of the fibrotic process, high local concentrations of inflammatory cell-derived trophic factors for mesenchymal cells direct their biological response $(3,4)$. However, even as the inflammatory response abates, the fibroproliferative process relentlessly continues, suggesting that intraalveolar mesenchymal cells may not require the continuous presence of exogenous signals to sustain the evolving fibroproliferative response.

An expanding body of experimental observations supports the concept that a number of fundamental phenotypic properties of mesenchymal cells can be stably altered. For example, stable changes in proliferative capacity have been observed in mesenchymal cells obtained from the affected organs of patients with atherosclerosis, progressive systemic sclerosis, idiopathic pulmonary fibrosis, and interstitial renal fibrosis (5-9). On the basis of this information we hypothesized that lung mesenchymal cells obtained from individuals dying with alveolar fibrosis after acute lung injury would stably manifest an intrinsically enhanced proliferative capacity. This issue was examined both in vitro and in vivo. The results indicated that in contrast to their normal counterparts, mesenchymal cells isolated from patients dying with acute lung injury were capable of undergoing cell division in defined medium lacking exogenously added peptide growth factors. Despite this altered proliferative phenotype, these cells did not display other features commonly associated with a transformed phenotype.

\section{Methods}

\section{Source of mesenchymal cells}

Six primary cultures of lung mesenchymal cells were developed from two groups of human lungs:

Acute lung injury $(n=3)$. This group consisted of the lungs from patients who died from respiratory failure 14,21 , and $30 \mathrm{~d}$ after disease onset. These patients met established criteria for the adult respiratory distress syndrome $(10,11)$ and represented a subset of a previously published cohort (3). Each lung specimen demonstrated the characteristic histological pattern of diffuse alveolar damage and intraalveolar fibrosis. 
Normal $(n=3)$. This group consisted of the unused lungs from single-lung transplantation donors. All were histologically normal.

\section{Preparation of mesenchymal cell cultures}

Mesenchymal cell primary cultures were developed by enzymatic and mechanical dispersion of lung tissue in a manner designed to optimize viability, purity, and yield. Lungs were processed as close to the time of death as possible (within 2 to $4 \mathrm{~h}$ ). An endotracheal tube was placed into a main-stem bronchus and the lungs were inflated with air (up to 5 $\mathrm{ml} / \mathrm{kg}$ body $\mathrm{wt}, \leq 30 \mathrm{~cm}$ hydrostatic pressure). The pulmonary arteries were cannulated and perfused with PBS $+0.02 \%$ EDTA (pH 7.4) until the left atrial effluent was clear. To remove all recoverable alveolar macrophages, sequential bronchoalveolar lavage was performed with PBS ( $30 \mathrm{~cm}$ of hydrostatic pressure, eight cycles), and the best perfused lobe was isolated and instilled with enzyme solution (Dulbecco's PBS containing [wt/vol]: 0.05\% trypsin, [Gibco Laboratories, Grand Island, NY], 0.008\% elastase, [ICN Biomedicals Inc., Costa Mesa, CA] and $0.005 \%$ DNAase I, [Sigma Chemical Co., St. Louis, $\mathrm{MO}]$ ) at $37^{\circ}, 30 \mathrm{~cm}$ of hydrostatic pressure for $30 \mathrm{~min}$.

After six cycles of bronchoalveolar lavage to recover intraalveolar mesenchymal cells, the parenchyma was sharp dissected from the airways, cut into 1-mm pieces, and suspended in DME (Sigma Chemical Co.) containing $0.05 \%$ (wt/vol) DNAase. The pieces were gently stirred $\left(30 \mathrm{~min}, 37^{\circ}\right)$ and filtered through nylon mesh $(100 \mu \mathrm{m}$, Tetco, Lancaster, NY) to produce a single-cell suspension. After hypotonic lysis of red blood cells $(0.2 \% \mathrm{NaCl}<10 \mathrm{~min})$, the single-cell suspension was transferred to standard medium (DME containing $10 \%$ calf serum, $100 \mathrm{U} / \mathrm{ml}$ penicillin, $100 \mathrm{mg} / \mathrm{ml}$ streptomycin, and $2.5 \mathrm{mg} / \mathrm{ml}$ amphotericin B) and seeded in tissue culture dishes (Falcon, Lincoln Park, $\mathrm{NJ})$ to allow mesenchymal cell attachment $\left(3 \mathrm{~h}, 37^{\circ}, 10 \% \mathrm{CO}_{2}\right)$. The supernatant fluid with unattached cells was replaced by standard medium and the cultures were continued. Medium was changed three times per week. In $10 \mathrm{~d}$ (cells from injured lungs) and 14-20 d (cells from normal lungs), the culture surface consisted of a densely packed monolayer of cells. Subcultivation was performed weekly at a split ratio of $1: 3$ as previously described (3). Aliquots of cells before each passage were frozen and stored under liquid nitrogen.

Since testing the hypothesis depends on the resultant in vitro cell population from lung-injured patients, reflecting in some reasonable manner the population of cells actually present in vivo, the population dynamics in primary culture were formally assessed. To accomplish this, parallel primary cultures were examined daily for cell number and DNA synthesis (below). No increase in cell number and virtually no DNA synthesis was observed for the first $3 \mathrm{~d}$ in primary culture. During days 4-10 of primary culture, cells underwent four to five population doublings until density arrested. During days 4-6, cells manifested a labeling index that increased from 60 to $90 \%$ per 24-h labeling period and a population doubling time that decreased from 29 to $\sim 21 \mathrm{~h}$. These values remained stable from days 7-10 of primary culture and thereafter through six subcultivations. On the basis of these data, the resultant in vitro cell population represented a reasonable first approximation to the original cell population in vivo.

\section{Characterization of recovered cells}

Cells were characterized as mesenchymal cells morphologically and by immunological criteria. In addition to routine phase-contrast microscopy, ultrastructural, immunofluorescence, and immunohistochemical examinations were carried out.

Preparation for electron microscopy. Samples of the mesenchymal cells cultured on coated beads (Cytodex 3; Pharmacia Inc., Piscataway, $\mathrm{NJ})$ from normal and injured lungs were prepared for study in the electron microscope (12-14). Medium above the beads covered with mesenchymal cells was reduced by aspiration. Glutaraldehyde (3\%) in White's saline was added to the cultured cells. Fixation continued at room temperature for $1 \mathrm{~h}$ and under refrigeration overnight. Supernatant fixative was aspirated the next morning, and cells were washed three times with Hanks' balanced salt solution. After washing, the cells were fixed again in $1 \%$ osmic acid in distilled water containing $1.5 \%$ potassium ferrocyanide for $1 \mathrm{~h}$ at $4^{\circ} \mathrm{C}$. The samples were dehydrated in a graded series of alcohol and embedded in Epon 812. Thin sections cut from the plastic blocks on an ultramicrotome were examined after staining with uranyl acetate and lead citrate to enhance contrast. Examination was carried out in an electron microscope (model 301; Philips Electronic Instruments, Inc., Mahwah, NJ).

Preparation for fluorescence microscopy. Fluorescence microscopy was used to identify the actin filaments in cultured mesenchymal cells from normal and injured lungs. Mesenchymal cells cultured on glass coverslips were fixed in methanol at $-10^{\circ} \mathrm{C}$ for $10 \mathrm{~min}$ followed by brief immersions of $3 \mathrm{~min}$ each in acetone at $-10^{\circ} \mathrm{C}$. Slides were washed by dipping in Hanks' balanced salt solution and allowed to air dry. Cells prepared in this manner could be stored in the refrigerator for later use but were ordinarily prepared immediately for fluorescence microscopy. After washing in the Hanks' balanced salt solution, the slides were transferred to a moist chamber. Rhodamine-phalloidin ( R415; Molecular Probes Inc., Eugene, OR) diluted in Hanks' balanced salt solution to $0.33 \mathrm{mM}$ was pipetted onto the slide. Incubation was continued for $30 \mathrm{~min}$ and mounted under coverslips in a solution of $P$-phenylinediamine-PBS-glycerine (15). The slides were studied under phase contrast and phase fluorescence in a photomicroscope (Zeiss, Oberkochen, Germany) equipped with an ultraviolet power source and appropriate barrier filters. The cells were observed and examined under epifluorescence optics and $60 \times$ and $100 \times$ planapo objectives.

Preparation for immunohistochemical analysis. To prepare samples for immunohistochemical studies, mesenchymal cells from injured and normal lungs were enzymatically dispersed (Trypsin $0.25 \%$; Gibco Laboratories Inc.; $37^{\circ}, 3 \mathrm{~min}$ ) and deposited on slides by cytocentrifugation after inactivation of trypsin with DME $+0.4 \%$ calf serum. Slides were air dried, dehydrated in graded alcohol, immersed in methanolic hydrogen peroxide $(0.6 \%$ [ $\mathrm{wt} / \mathrm{vol}$ ] to block endogenous peroxidase activity), rehydrated in graded alcohols, and placed in PBS (pH 7.4, $10 \mathrm{~min}$ ). After primary antibody application, the tissue was incubated in a moisture chamber $\left(16 \mathrm{~h}, 4^{\circ}\right)$. Subsequent antibody bridge assembly using the avidin-biotin peroxidase complex procedure was carried out as described (16). Chromogenic development was accomplished by immersion of the cells in 3,3' diaminobenzidine hydrochloride solution containing $0.003 \%$ hydrogen peroxide ( $<10 \mathrm{~min}$ ), dipping in $0.125 \%$ (wt/ vol) osmium tetroxide, and rinsing in water. The following primary antibodies were used to characterize the lung cell isolates: antivimentin (V9; BioGenex Labs., San Ramon, CA), antimuscle-specific actin (HHF-35; Enzo Biochem, New York), antidesmin (DEB-5, BioGenex Labs.), anticytokeratin cocktail (AE1/ AE3; Hybitech, Indianapolis, IN; Cam 5.2; Becton Dickinson, Mountain View, CA; MAK 6; Triton Diagnostics, Alameda, CA) antiepithelial membrane antigen (E 29; DakoPatts, Carpinteria, CA), antifactor VIII (Accurate Chem. \& Sci. Corp., Westbury, NY), and anti-CD45 (AHN-12, kindly provided by Dr. K. Skubitz [17]). The binding of ulex europaeus lectin ("ulex"; Accurate Chem. \& Sci. Corp.) to each isolate was also evaluated.

\section{Proliferative capacity of lung mesenchymal cells in defined medium}

Growth. A defined basal medium (defined medium; $F_{12}$ containing 25 $\mathrm{mM}$ Hepes, $\mathrm{pH} 7.4,0.1 \mathrm{mg} / \mathrm{ml}$ bovine serum albumin, $10 \mu \mathrm{g} / \mathrm{ml} \mathrm{Fe}-$ transferrin, $10^{-8} \mathrm{M}$, selenium, $3 \times 10^{-6} \mathrm{M}$ linoleic acid), was used to assess mesenchymal cell proliferative capacity. The defined medium did not allow more than a $5 \%$ increase in cell number per day in a well-characterized normal lung fibroblast strain (ATCC, CCL 210).

To examine cell division, mesenchymal cells from injured and normal lungs were enzymatically removed from tissue culture dishes (trypsin $0.25 \%, 37^{\circ} \mathrm{C}, 3 \mathrm{~min}$ ) and centrifuged $(600 \mathrm{~g}, 10 \mathrm{~min})$ after the inactivation of trypsin with DME containing $0.4 \%$ calf serum. Cells were resuspended in this medium and seeded in tissue culture dishes. After $2 \mathrm{~d}$ of culture $\left(37^{\circ}, 90 \%\right.$ air, $\left.10 \% \mathrm{CO}_{2}\right)$, cells were rinsed three 
times with defined medium and cultures were continued in that medium with no exogenous growth factors for up to $9 \mathrm{~d}$. Preliminary growth studies indicated that after $2 \mathrm{~d}$ in DME $+0.4 \%$ serum the number of mesenchymal cells from injured lungs was $>50 \%$ higher than that from normal lungs. Therefore, to achieve equivalent cell densities at the time cultures were switched to defined medium (see below); the mesenchymal cells from injured lungs were seeded at a lower density $\left(4.0 \times 10^{3}\right.$ cells $\left./ \mathrm{cm}^{2}\right)$ than the normal mesenchymal cells $\left(6.0 \times 10^{3}\right.$ cells $\left./ \mathrm{cm}^{2}\right)$. At selected times, cells were detached from culture dishes with trypsin/EDTA and their growth assessed by direct cell counts using an automated particle counter (Coulter Corp. Hialeah, FL).

DNA synthesis. To quantify the proportion of cells synthesizing DNA in defined medium, autoradiography of cells cultured with $\left[{ }^{3} \mathrm{H}\right.$ ] thymidine was performed as described by Baserga and Malamud (18). Labeling was carried out for a 16-h period between the fourth and fifth days of culture in defined medium. During that interval, cultures had reached $\sim 50 \%$ of their saturation density. Autoradiograms were exposed for $5 \mathrm{~d}$, and slides were counter-stained with hematoxylin and eosin.

Mesenchymal cell responsiveness to growth factors. To assess their responsiveness to growth factors, lung mesenchymal cells were cultivated in defined medium supplemented singly or in combination with well-characterized mesenchymal cell growth factors as previously described ( 3 ). For these studies platelet-derived growth factor (PDGF) ${ }^{\prime}$ (1-100 pM), epidermal growth factor (EGF) (1-100 pM), or insulin (1-100 nM) was used. Briefly, cells enzymatically removed from culture dishes were seeded at $6.0 \times 10^{3}$ cells $/ \mathrm{cm}^{2}$ in DME $+0.4 \%$ serum. After $3 \mathrm{~d}$, cells were rinsed three times with defined medium and cultures continued in that medium for $1 \mathrm{~d}$. Cells were rinsed one additional time with defined medium and cultures were continued for $3 \mathrm{~d}$ in defined medium with or without growth factors as indicated. Cells were enumerated using an electronic particle counter (see above). Defined medium served as a negative control and standard medium served as a positive control.

\section{Examination of mesenchymal cells for expression of the immediate early cell division cycle genes c-fos and c-jun}

To determine if the mesenchymal cells recovered from injured lung displayed the pattern of immediate early cell division cycle gene expression characteristic of proliferating fibroblasts, Northern blot analysis for $\mathrm{c}$-fos and c-jun was performed. Actively proliferating mesenchymal cells $\left(5 \times 10^{6}\right)$ from injured lungs were cultured in defined medium. RNA was extracted as described by Chomczynski and Sacchi (19), electrophoresed in $1.0 \%$ formaldehyde-agarose gels, transferred to $\mathrm{Ni}$ tran, and hybridized with probes for c-fos (20) and c-jun (21), labeled with ${ }^{32} \mathrm{P}$ by random priming to a specific activity of 0.8 to $1.2 \times 10^{9}$ $\mathrm{cpm} / \mu \mathrm{g}$. Controls included parallel cultures of mesenchymal cells from normal lungs cultured in defined medium (nonproliferating, negative control) or those pulsed with $10 \%$ serum ( $45 \mathrm{~min}$, positive controls).

\section{Examination of mesenchymal cells for release of growth regulatory peptides \\ Production of mesenchymal cell supernates. Mesenchymal cells were} cultivated in standard medium until confluent. Cells were rinsed three times with defined medium with cultures continued in this medium for 4,24 , and $48 \mathrm{~h}$. The resultant mesenchymal cell-conditioned medium was centrifuged $(600 \mathrm{~g}, 10 \mathrm{~min})$ and stored at $-70^{\circ}$ until assay. Each supernate was analyzed for its ability to influence mesenchymal cell replication (see below).

Ability of mesenchymal cell supernates to influence cell replication. To examine this issue, a cell replication assay was performed as de-

1. Abbreviations used in this paper: EGF, epidermal growth factor; PDGF, platelet-derived growth factor; SCID, severe combined immunodeficiency. scribed (3). Mesenchymal cell supernates served as the test sample The rationale was to use an assay system that could detect both established as well as novel autocrine growth factors for mesenchymal cells. The target lung fibroblast chosen (ATCC, CCL 210) manifested a high level of growth factor responsiveness as well as minimal replication in the absence of exogenous trophic signals.

\section{Examination of mesenchymal cells for a transformed phenotype}

Three biological properties were examined to assess the possibility that the mesenchymal cells isolated from acutely injured lungs were transformed: karyotype analysis; senescence/immortality in vitro; and ability to proliferate in mice with severe combined immunodeficiency (SCID).

Karyotype. To examine the distribution and number of chromosomes in each isolate, routine karyotyping was carried out as described by Priest (22). For each isolate $>20$ metaphases were examined using $\mathrm{G}$ banding.

In vitro lifespan. Mesenchymal cells were subcultivated in standard medium (see above) with subsequent growth rate and phase-contrast morphology assessed.

Growth after intraperitoneal injection into immunodeficient mice. To characterize the growth properties of the recovered mesenchymal cells in vivo, the ability of each strain to proliferate after injection into the peritoneal cavity of immunodeficient mice was assessed (23). Female CB-17 mice with SCID were purchased from Taconic Farms, Inc. (Germantown, NY) and used at 9 wk of age. Mice were maintained in microisolator cages in a specific pathogen-free colony under laminar flow conditions. Mice were given antibiotic-supplemented water and food ad lib. Mesenchymal cells $\left(10^{6}\right.$ in defined medium from normal individuals or patients with acute lung injury were injected in the peritoneal cavity of SCID mice ( $n=5$ per group). Mice were monitored twice weekly for clinical health, survival, and the development of ascites fluid or other evidence of tumor metastases. Macroscopic postmortem review, examination of routine histological sections (hematoxylin and eosin), and immunohistochemical analysis of snap-frozen organs (anti-human HLA antibodies; Accurate Chem. \& Sci. Corp.) served as criteria for in vivo dissemination.

\section{Results}

Characterization of recovered lung cells. Cells cultured from normal $(n=3)$ and acutely injured lungs $(n=3)$ were examined morphologically, by immunofluorescence microscopy, and immunohistochemically. Phase-contrast microscopy of each isolate demonstrated the typical spindle shaped appearance of lung fibroblasts (Fig. $1 A$ ). Ultrastructurally, the characteristic features of myofibroblasts were observed. Flattened nuclei were surrounded by stretched out cytoplasm containing rough endoplasmic reticulum, mitochondria, occasional nonspecific granules, and masses of filaments. Diameters of individual microfilaments were $50-60 \mathrm{~nm}$, suggestive of actin filaments. In some cells the filaments were concentrated along the membrane surface adjacent to the bead. There were no apparent differences observed in sections from normal and injured lung cell cultures.

Examination of cultured mesenchymal cells stained with rhodamine-phalloidin in the fluorescence microscope revealed large masses of stained filaments. In cells from normal lungs the filaments were primarily concentrated in long bundles resembling stress fibers (Fig. $1 B$ ). The actin filaments selectively stained by rhodamine-phalloidin in cells from injured lung tissue were also in bundles. However, the bundles were shorter and often spindle shaped. Background filaments were less orga- 

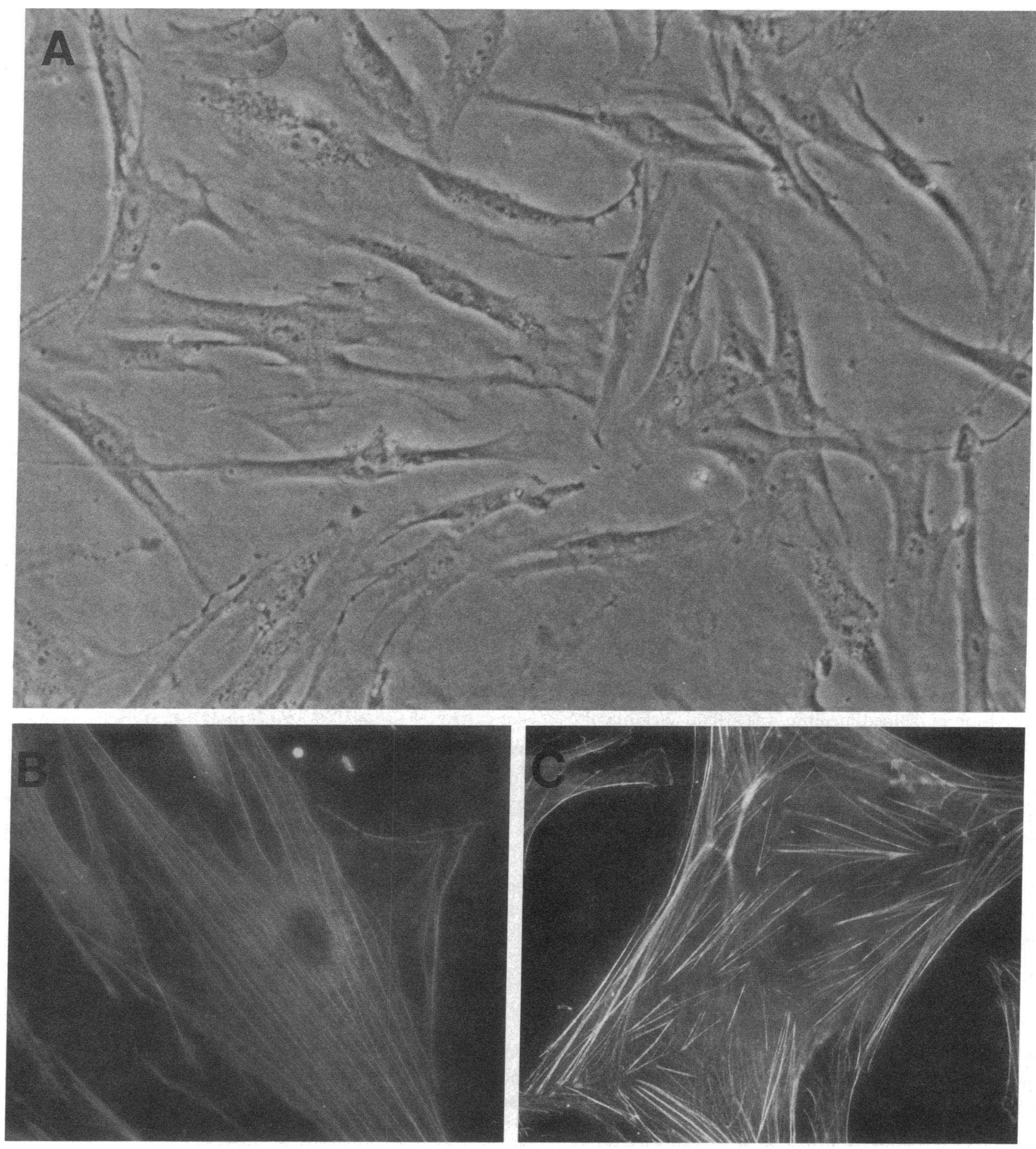

Figure 1. Morphology of lung cell isolates. $(A)$ Phase-contrast appearance of a representative isolate from the lungs of a patient dying with acute lung injury. The appearance was similar to cells isolated from normal lungs. Fluorescence microscopic appearance of mesenchymal cells stained with rhodamine-phalloidin. Shown is the actin filament array of a representative $(B)$ normal and $(C)$ lung-injured isolate.

nized and often appeared ragged compared with the smooth bundles in cells from normal lung (Fig. $1 C$ ).

Immunohistochemical studies indicated the cells were positive for determinants characteristic of mesenchymal cells (vimentin, muscle specific actin, desmin) but were negative for endothelial cell determinants (Factor VIII, Ulex), epithelial cell determinants (cytokeratin, epithelial membrane antigen) and a leukocyte determinant (CD45).
Proliferative state of lung mesenchymal cells. Cultivation of each lung mesenchymal cell strain in defined medium lacking exogenous peptide growth factors revealed a striking difference between those derived from normal lungs and those from acutely injured lungs (Fig. 2). As expected, the replication rate of mesenchymal cells from normal lungs slowed greatly after cultivation for $2 \mathrm{~d}$ in medium containing a low concentration of serum (DME $+0.4 \%$ calf serum). With subsequent cultiva- 


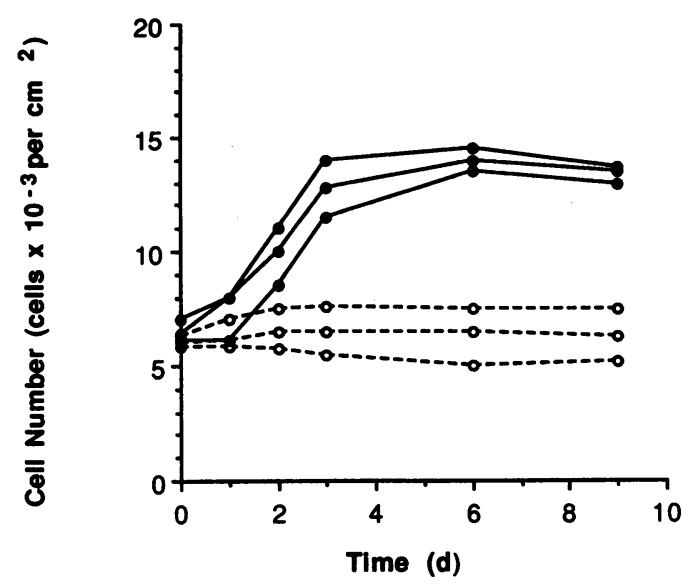

Figure 2. Growth of lung mesenchymal cells. Each isolate was cultured in defined medium lacking exogenous growth factors. Shown is cell number as a function of time in culture for isolates derived from normal (- - ) and acutely injured ( - ) lungs.

tion in defined medium lacking exogenous peptide growth factors, the mesenchymal cells from normal lungs essentially failed to divide $\left(6,475 \pm 468\right.$ cells $/ \mathrm{cm}^{2}$ on day 0 compared with $6,866 \pm 950$ cells $/ \mathrm{cm}^{2}$ on day $\left.6, P=0.56\right)$. In marked contrast, mesenchymal cells from acutely injured lungs doubled within 3 to $6 \mathrm{~d}$, reaching maximum density by $6 \mathrm{~d}(6,828 \pm 258$ cells / $\mathrm{cm}^{2}$ on day 0 compared with $14,617 \pm 480$ cells $/ \mathrm{cm}^{2}$ on day 6 , $P<0.001$ ). Of note, this enhanced proliferative state permitting cell division in defined medium was stable. Each mesenchymal cell strain prepared from injured lungs maintained this enhanced proliferative phenotype for at least five subcultivations (Fig. 3).

In accord with the results of direct cell counts, cultured mesenchymal cells from injured lungs were actively synthesizing DNA (Fig. 4). A representative assessment of each mesenchymal cell strain was obtained by labeling cells for $16 \mathrm{~h}$ between the fourth and fifth day of culture in defined medium. Although the percentage of cells obtained from normal lungs that synthesized DNA during the labeling interval was always $<4 \%($ mean $\pm \mathrm{SD}=1.2 \pm 0.8)$, the values for mesenchymal cells derived from injured lungs ranged from 25 to $35 \%$. The close concordance between the estimated DNA synthesis and the resultant increase in cell numbers for each cell strain suggested that nearly all labeling observed was directed toward cell division.

Mesenchymal cell replication in response to peptide growth factors. Although capable of sustained division in defined medium lacking peptide growth factors, mesenchymal cells from injured lungs retained a normal profile of growth factor responsiveness (Fig. 5). Similar to mesenchymal cells from normal lungs, those from acutely injured lungs grew well in response to PDGF and EGF individually, while showing little or no response to insulin as a sole factor. The combination of PDGF, EGF, and insulin stimulated the replication of all strains of mesenchymal cells in a synergistic fashion. Omission of PDGF, EGF, or insulin resulted in a 38,27 , or $16 \%$ decrease, respectively, in the number of cells (data not shown). The maximum mesenchymal cell growth response to the optimal combination of all three growth factors ranged from 60 to $68 \%$ of the re-
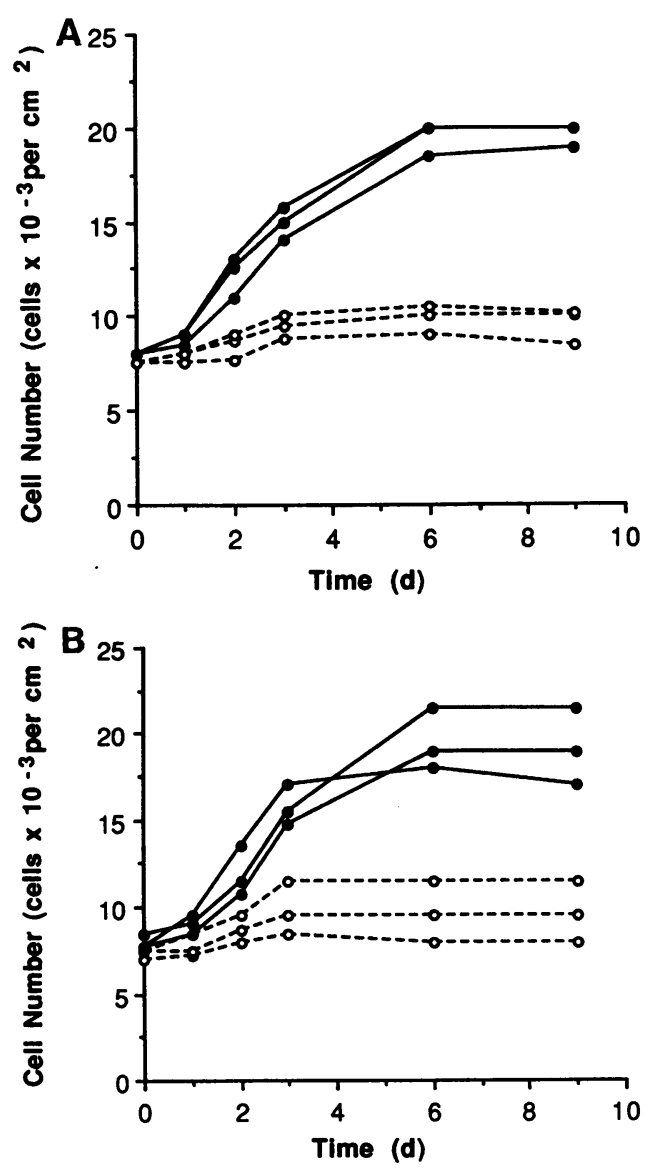

Figure 3. Stability of lung mesenchymal cell proliferative phenotype. Shown is cell number as a function of time in culture in defined medium lacking exogenous growth factors after the $(A)$ third and $(B)$ fifth subcultivation. Symbols for isolates are as in the legend to Fig. 2.

sponse to standard medium containing $10 \%$ serum. Cell strains were virtually indistinguishable in this regard.

Immediate early cell division cycle gene expression: c-fos and $c$-jun. Consistent with the observed proliferative phenotype, all three mesenchymal cell strains demonstrated constitutive expression of both c-fos and c-jun when cultured in defined medium (Fig. 6). Cells prepared from normal lungs manifested no detectable c-fos or c-jun expression in defined medium but manifested high level expression after a 45-min exposure to medium containing $10 \%$ serum (not shown). Each strain showed a similar pattern of immediate early gene expression for up to five subcultivations.

Influence of mesenchymal cell supernates on the growth of nonreplicating lung fibroblasts. The unique growth properties of mesenchymal cells obtained from injured lungs prompted an examination of autocrine growth factor release by these cells. The growth-promoting properties of mesenchymal cell supernates from each strain were assessed in a standard bioassay system using an established normal diploid lung fibroblast strain as the target cell. These target cells were used because of their high level of responsiveness to established growth factors. Supernates from both normal and lung-injured mesenchymal cells failed to reveal significant growth promoting bioactivity. Addition of exogenous growth factors (PDGF, EGF, insulin) 


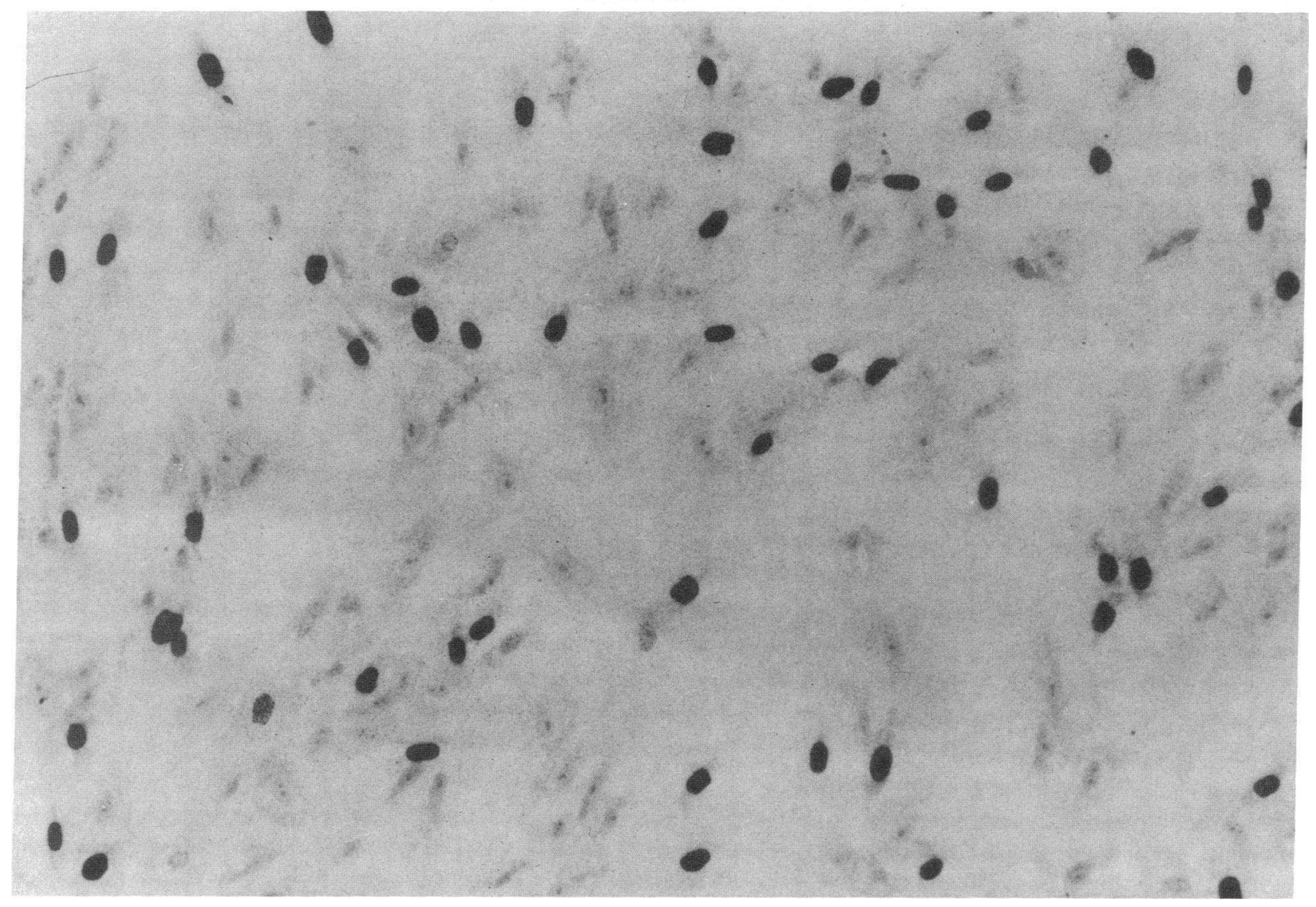

Figure 4. DNA synthesis by lung mesenchymal cells. Each isolate of lung mesenchymal cells (second subcultivation) was cultured as in Fig. 2. During the fifth day in culture, nuclei synthesizing DNA were labeled $(16 \mathrm{~h})$ with $\left[{ }^{3} \mathrm{H}\right]$ thymidine, fixed, overlayed with photographic emulsion, exposed for $5 \mathrm{~d}$, and developed. Shown is a representative example of results obtained with mesenchymal cells isolated from acutely injured lungs (Wright's stain).

to each supernate yielded the expected growth response, suggesting that inhibitors in the test supernates were not masking the presence of autocrine factors.

Assessment of lung mesenchymal cells for a transformed phenotype. Each mesenchymal cell strain isolated from injured lungs was diploid, displaying a normal number of chromosomes. In addition, no strain manifested immortality in culture, becoming senescent after the 9th or 10th subcultivation. Consistent with this behavior, no strain of lung mesenchymal cells from injured lungs proliferated or disseminated after injection into the peritoneal cavity of SCID mice. Histological and immunocytochemical examination failed to reveal the presence of human mesenchymal cells in the abdominal or thoracic viscera.

\section{Discussion}

Mesenchymal cells constitute an integral part of the highly coordinated cellular response required for tissue repair after injury. The present study indicates that lung mesenchymal cells obtained from patients dying with acute lung injury have a unique in vitro proliferative phenotype. In contrast to mesenchymal cells obtained from histologically normal lungs, cells from injured lungs are capable of sustained proliferation in the absence of exogenous peptide growth factors. In accord with their proliferative phenotype, mesenchymal cells from injured lungs constitutively express the immediate early genes, c-fos and c-jun. This proliferative phenotype is stable for at least five subcultivations and does not appear to depend on autocrine release of trophic factors. Despite their enhanced proliferative capacity, mesenchymal cells from injured lungs are not transformed. They remain diploid with a normal number of chromosomes, manifest a normal proliferative response to exogenous peptide growth factors, demonstrate a finite lifespan in vitro, and do not form tumors in the highly permissive SCID mouse system.

One concept of the biological response to tissue injury is that paracrine signals derived from inflammatory cells direct mesenchymal cell function. According to this paradigm, mesenchymal cell migration, proliferation, and connective tissue biosynthesis are upregulated when trophic factors are present and are downregulated by inhibitory factors. As inflammation subsides, these mesenchymal cell functions relax to their normal homeostatic level. If for some reason, such as infection of the injured tissue, inflammation persists, the continued presence of inflammatory cell-derived signals lead to disregulation of mesenchymal cell function and a fibroproliferative response.

Although this formulation fits reasonably well when effective tissue repair occurs, several observations suggest this may not be the case when a fibroproliferative response ensues. Our 


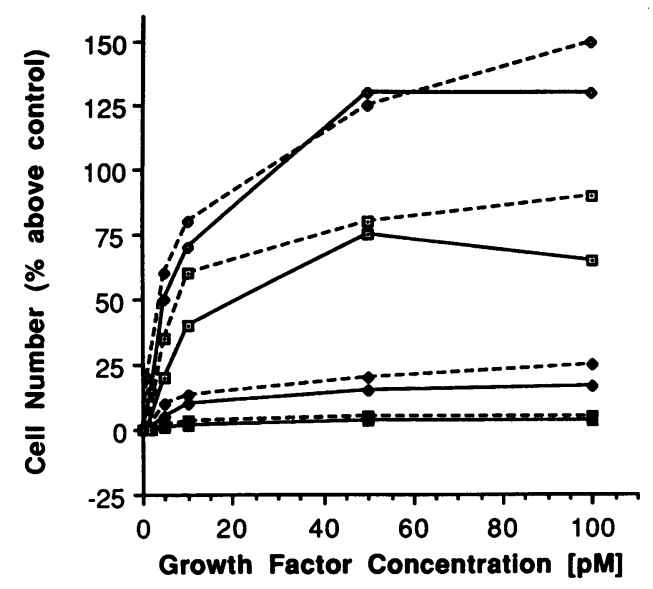

Figure 5. Mesenchymal cell responsiveness to growth factors. Each isolate was seeded and cultured in DME $+0.4 \%$ calf serum for $3 \mathrm{~d}$. Cells were cultured in defined medium for one additional day with cultures continued in the presence of well-characterized growth factors, singly or in combination. Shown is the mean growth response for mesenchymal cells from normal (- - ) and acutely injured lungs $(-)$ in response to insulin, $100 \mathrm{nM}(\bullet)$; EGF, $100 \mathrm{pM}(\diamond)$; PDGF, $100 \mathrm{pM}(\square)$; and the combination of all three growth factors $(\diamond)$.

data indicate that fibroblasts recovered from the lungs of patients 2-4 wk after acute lung injury manifest an increased proliferative capacity. This is a time when histological evidence of inflammation is minimal $(5,9)$. Independence of this phenomenon from persistently present inflammatory cell-derived signals is established by the stability of the observed proliferative phenotype for five subcultivations. In addition to our findings, similar ex vivo stability of altered mesenchymal cell phenotypic properties has been observed in smooth muscle cells derived from the intima of atherosclerotic plaques $(7,9)$, skin fibroblasts derived from patients with progressive systemic sclerosis (6), renal fibroblasts derived from patients with interstitial fibrosis (8), and lung fibroblasts cultured from patients with pulmonary fibrosis (5). Examination of plaque-derived smooth muscle cells for clonality, protooncogene expression pattern, and transforming potential of isolated DNA has been carried out $(7,9,24)$. These studies indicate that plaque-derived mesenchymal cells are mono/oligoclonal, have increased expression of $c-m y c$, and contain transforming activity. On the basis of these results a number of investigators have suggested that a somatic mutation underlies the pathological process, although definitive proof identifying the altered locus is lacking. Of note, similar to our findings, plaque-derived smooth muscle cells did not directly form tumors in nude mice (7). Collectively these results are consistent with the concept that, in fibroproliferative disorders, stable alteration of mesenchymal cell phenotype can occur. Whether it occurs by epigenetic means or by somatic mutation remains an open question.

There are at least three possible explanations of our results that demonstrate growth factor-independent proliferation of myofibroblasts isolated from patients dying with acute lung injury: that a large proportion of resident mesenchymal cells have been altered by signals in the injured lung, conferring upon them a stable differentiated state manifested by an intrinsically enhanced proliferative capacity; that selective expansion of a normally resident mesenchymal cell has occurred in vivo,

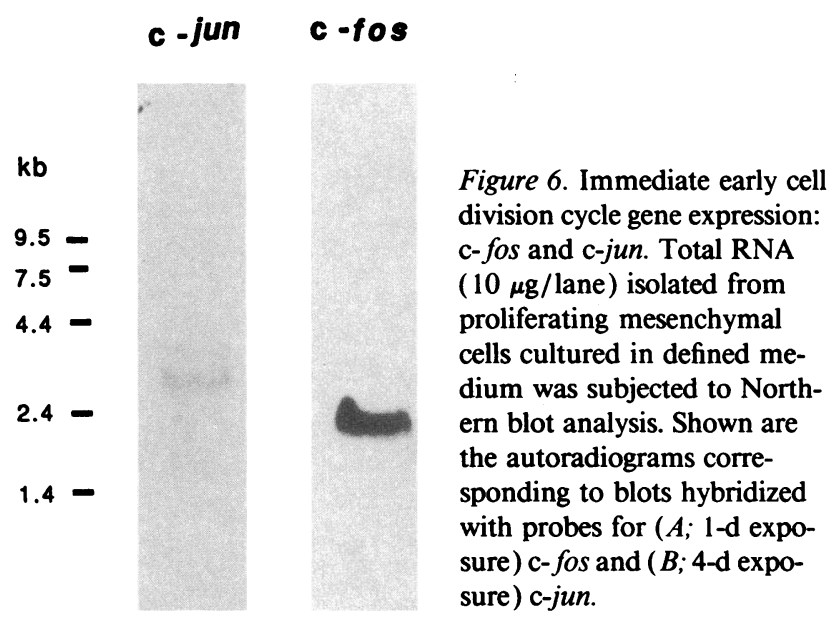

making it the numerically most important constituent of the injured lung; and that the results are representative of only a small subpopulation of the in vivo cell population that enjoy a selective advantage in culture.

Distinguishing between the first two explanations is not possible at present, since antibodies and nucleotide probes that can discriminate between mesenchymal cells derived from normal lungs and acutely injured lungs are not available for in situ analysis of tissue specimens. Our results serve to highlight the need to develop such reagents. Although the third explanation is formally possible, several points argue against it as the sole explanation of our results.

First, all primary culture cells from lung injury patients underwent at most four to five population doublings to reach confluence in $10 \mathrm{~d}$. During this time interval, virtually no proliferation was observed during the first $3 \mathrm{~d}$ in culture, leaving only $7 \mathrm{~d}$ to undergo four to five population doublings. Since the population doubling time of the resultant myofibroblast population under identical culture conditions was $\sim 21 \mathrm{~h}$, at most a two- to fourfold enrichment of a highly proliferative subpopulation could have occurred. This is probably an overestimate, since mesenchymal cells derived from "normal lungs" (which yielded confluent monolayers after 14-20 d) manifested a stable doubling time of 24-26 h. Assuming that these normal fibroblasts are a reasonable first approximation to the cells being overgrown, very little in vitro enrichment could have occurred during primary culture.

Second, the labeling index of the cells from lung-injured patients cultured in defined medium is consistently $25-35 \%$ and the population doubling time is $3-4 \mathrm{~d}$ for up to six subcultivations. If continuous enrichment for a highly proliferative subpopulation were occurring in vitro, these values should not remain stable.

Finally, cells from patients with lung injury when cultivated in serum have a population doubling time of $21 \mathrm{~h}$ and a labeling index of $85-90 \%$, values that remain stable from the first to sixth subcultivation. If enrichment for a highly proliferative subpopulation were occurring in vitro these values should also change.

Our observations suggest a more comprehensive view of the fibroproliferative response to tissue injury is in order. This view should acknowledge the mesenchymal cell as more than simply 
a passive participant in the process. Available information suggests that when a fibroproliferative response occurs in the lung, the kidney, the skin, or in a blood vessel, mesenchymal cells with an enhanced proliferative phenotype are present. This proliferative phenotype allows the participating mesenchymal cells to continue the fibroproliferative response without further exogenous signaling. It remains a challenge for future investigations to elucidate the molecular basis of this unique mesenchymal cell phenotype and to define the exogenous signals that induce its formation.

\section{Acknowledgments}

The authors would like to thank Dr. Betsy Hirsh for karyotype analysis; Debra Moore for assistance with autoradiography; Lan Chiang and Dr. Clifford Steer for assistance with Northern blot analysis; and Funmi Thompson, Deborah Sowells, and Elaina Davis for preparation of the manuscript.

\section{References}

1. Fukuda, Y., M. Ishizami, Y. Masuda, G. Kimora, O. Kawanami, and Y. Masugi. 1987. The role of intraalveolar fibrosis in the process of pulmonary structural remodeling in patients with diffuse alveolar damage. Am. J. Pathol. 126:171-182.

2. Katzenstein, A. A., J. L. Myers, and M. U. Mazur. 1986. Acute interstitial pneumonia: a clinicopathologic, ultrastructural, and cell kinetic study. $\mathrm{Am}$. J. Surg. Pathol. 10:256-267.

3. Snyder, L. S., M. I. Hertz, M. S. Peterson, K. R. Harmon, W. A. Marinelli, C. A. Henke, J. R. Greenheck, B. Chen, and P. B. Bitterman. 1991. Acute lung injury. Pathogenesis of intraalveolar fibrosis. J. Clin. Invest. 88:663-673.

4. Henke, C. A., V. Fiegel, M. Peterson, D. Knighton, J. McCarthy, and P. B. Bitterman. 1991. Identification and partial characterization of angiogenesis bioactivity in the lower respiratory tract after acute lung injury. J. Clin. Invest. 88:1386-1395.

5. Jordana, M., J. Schulman, C. McSharry, L. B. Irving, M. T. Newhouse, G. Jordana, and J. Gauldie. 1988. Heterogeneous proliferative characteristics of human adult lung fibroblasts lines and clonally derived fibroblasts from control and fibrotic tissue. Am. Rev. Respir. Dis. 137:579-584.

6. Leroy, E. C. 1974. Increased collagen-synthesis by scleroderma skin fibroblasts in vitro. A possible defect in the regulation of activation of the scleroderma fibroblast. J. Clin. Invest. 54:880-889.

7. Parkes, J. L., R. R. Cardell, F. C. Hubbard, Jr., D. Hubbard, A. Meltzer, and A. Penn. 1991. Cultured human atherosclerotic plaque smooth muscle cells retain transforming potential and display enhanced expression of the myc protooncogene. Am. J. Pathol. 138:765-775.

8. Rodemann, H. P., and G. A. Muëller. 1990. Abnormal growth and clonal proliferation of fibroblasts derived from kidneys with interstitial fibrosis. Proc. Soc. Exp. Biol. Med. 195:57-63.

9. Yoshida, Y., M. Mitsumata, T. Yamane, M. Tomikawa, and K. Nishida. 1988. Morphology and increased growth rate of atherosclerotic intimal smoothmuscle cells. Arch. Pathol. Lab. Med. 112:987-996.

10. Fowler, A. A., R. F. Hamman, G. O. Zerbe, K. N. Benson, and T. M. Hyers. 1985. Adult respiratory distress syndrome: prognosis after onset. Am. Rev. Respir. Dis. 132:472-478.

11. Petty, T. L., and D. G. Ashbaugh. 1971. The adult respiratory distress syndrome. Chest. 60:233-239.

12. Gerrard, J. M., D. R. Phillips, G. H. R. Rao, E. F. Plow, D. A. Ealz, R. Ross, L. A. Harker, and J. G. White. 1980. Biochemical studies of two patients with the gray platelet syndrome-selective deficiency of platelet alpha granules. J. Clin. Invest. 66:102-109.

13. White, J. G. 1968. Fine structural alterations induced in platelets by adenosine disphosphate. Blood. 31:604-622.

14. White, J. G. 1972. Interaction of membrane systems in blood platelets. Am. J. Pathol. 66:295-312.

15. White, J. G. 1981. A simple method of reducing the fading of immunofluorescence during microscopy. J. Immunol. Methods. 43:349.

16. Hsu, S. M., L. Raine, and H. J. Fanger. 1981. Use of avidin-biotin-peroxidase complex $(\mathrm{ABC})$ in immunoperoxidase techniques: a comparison between ABC and unlabeled antibody (PAP) procedures. J. Histochem. Cytochem. 29:577-580.

17. Skubitz, K. M., D. D. Ehresmann, and T. P. Ducker. 1991. Characterization of human neutrophil ecto-protein kinase activity released by kinase substrates. J. Immunol. 147:638-650.

18. Baserga, R., and D. Malamud. 1969. Autoradiography: Techniques and Applications. Harper and Row, New York/Evanston/London.

19. Chomzcynski, P., and N. Sacchi. 1987. Single-step method of RNA isolation by acid guanidinium thiocyanate-phenol-chloroform extraction. Anal. Biochem. 162:156-159.

20. Miller, A. D., T. Curran, and I. M. Verma. 1984. c-fos Protein can induce cellular transformation: a novel mechanism of activation of a cellular oncogene. Cell. 36:51-60.

21. Angel, P., E. A. Allegretto, S. T. Okino, K. Hatori, W. J. Boyle, T. Hunter, and $M$. Karin. 1988. Onocogene jun encodes a sequence specific trans-activator similar to AP-1. Nature (Lond.). 332:166-171.

22. Priest, G. 1977. Medical Cytogenetics and Cell Culture. Lea and Febiger, Philadelphia.

23. Camel-Reid, S., and J. I. Dick. 1989. A model of human acute lymphoblastic leukemia in immune-deficient SCID mice. Science (Wash. DC). 246:15571600 .

24. Benditt, E. P., and J. M. Benditt. 1973. Evidence for monoclonal origin of human atherosclerotic plaques. Proc. Natl. Acad. Sci. USA. 70:1753-1756. 